\title{
On the geometrical and electronic structure of an ultra-thin crystalline silica film grown on Mo(112).
}

\author{
S. Kaya ${ }^{1}$, M. Baron ${ }^{1}$, D. Stacchiola ${ }^{1}$, J. Weissenrieder ${ }^{1}$, S. Shaikhutdinov ${ }^{1 *}$, \\ T. K. Todorova ${ }^{2}$, M. Sierka ${ }^{2 *}$, J. Sauer ${ }^{2}$, H.-J. Freund ${ }^{1}$ \\ ${ }^{1}$ Fritz-Haber-Institut der Max-Planck-Gesellschaft, Faradayweg 4-6, \\ 14195 Berlin, Germany \\ ${ }^{2}$ Humboldt-Universität zu Berlin, Institut für Chemie, Unter den Linden 6, \\ 10099 Berlin, Germany
}

\begin{abstract}
The atomic structure of a well-ordered silica film grown on a Mo(112) single crystal substrate is discussed in detail using the experimental and theoretical results available to date. New photoelectron spectroscopy results using synchrotron radiation and ultraviolet spectroscopy data are presented. The analysis unambiguously shows that the ultra-thin silica film consists of a two-dimensional network of corner sharing $\left[\mathrm{SiO}_{4}\right]$ tetrahedra chemisorbed on the unreconstructed $\mathrm{Mo}(112)$ surface. The review also highlights the important role of theoretical calculations in the determination of the atomic structure of the silica films and in interpretation of experimental data.
\end{abstract}

Keywords: thin oxide films; oxide surfaces; silica; scanning tunneling microscopy; vibrational spectroscopy; photoelectron spectroscopy; density functional theory *Corresponding authors: marek.sierka@chemie.hu-berlin.de; shaikhutdinov@fhi-berlin.mpg.de

\section{Introduction.}


In recent years, thin oxide films have received much interest due to their potential use in many technological applications such as insulating layers in integrated circuits, protective films against corrosion, and supports for metal nanoparticles in sensors and catalysts. When grown on metal substrates, oxide films are well suited for studying electronic and chemical properties by a variety of surface sensitive techniques. This "surface science" approach has been proven to be very efficient for determining the atomic structure of many oxide surfaces (see [1-12] and references therein).

Silica $\left(\mathrm{SiO}_{2}\right)$ is one of the most important oxides in advanced technology. Although the first attempts to synthesize crystalline silica films can be traced back to the early 90's $[13,14]$, the preparation of well-ordered thin silica films was reported only recently by Schroeder et al [15-18] using a Mo(112) substrate, who basically modified a recipe originally applied to the $\operatorname{Mo}(100)$ and $\operatorname{Mo}(110)$ substrates by Goodman and coworkers $[13,14]$. The film exhibited a $\mathrm{c}(2 \times 2)$ diffraction pattern with respect to $\mathrm{Mo}(112)$, and showed at least two oxygen species in X-ray photoelectron spectra (XPS) and a strong phonon absorption band centered at $1048 \mathrm{~cm}^{-1}$ observed by infrared reflection absorption spectroscopy (IRAS). During the last five years there have been numerous experimental and theoretical efforts in order to elucidate the atomic structure of these films and employ their use as model supports for metal (Pd, Au, Ag) particles [19-45].

Based on attenuation of the substrate signals in Auger electron spectroscopy (AES) and angular-resolved XPS spectra, Schroeder et al. [15] came to the conclusion that the well ordered films are 5-8 $\AA$ in thickness. Based on AES data Kim et al. [24] also reported on the film thickness of 7-8 A. Later the same group, using another preparation, concluded from the AES results that the film is only $3 \AA$ in thickness [28].

In the first structural model proposed by Schroeder et al. [18], the film was bonded to the Mo substrate through three oxygen atoms sitting in pseudo three-fold hollow sites formed between protruding and trough Mo atoms, forming rows on the $\operatorname{Mo}(112)$ surface along the $[\overline{1} \overline{1} 1]$ direction. These sites were suggested as the most stable for oxygen chemisorption on Mo surfaces. Assuming that a $\left[\mathrm{SiO}_{4}\right]$ tetrahedron is the building unit for the silica films, the proposed structure involved a maximum coordination of oxygen atoms to the Mo surface, i.e. three $\mathrm{O}$ atoms of each tetrahedra 
were bonded to the Mo atoms. In this model, the forth ("apical") oxygen atom of each tetrahedra (which remains uncoordinated in the case of a monolayer silica film) must be stabilized either by hydrogen, thus forming silanol ( $\mathrm{Si}-\mathrm{OH})$ groups, or shared with further silica layers. Since neither silanol nor hydroxyl species were observed on these films [18], this implied that the silica film was of several layers in thickness.

Following this model and assuming that the film is three layers of $\mathrm{SiO}_{2}$, Ricci and Pacchioni [27] proposed a $\beta$-cristobalite derived structure as thermodynamically the most stable. In particular, formation of two-membered rings were invoked to explain a weak vibration band around $795 \mathrm{~cm}^{-1}$ experimentally observed by Schroeder et al [18].

However, Chen et al. [28], analyzing high resolution electron energy loss spectra (HREELS) of the silica films and various $\mathrm{Si}$ and Mo oxide compounds available in the literature, have suggested that the most prominent absorption band at $1048 \mathrm{~cm}^{-1}$ observed on the film must be assigned to the Si-O-Mo vibration. The absence of the band in the $1150-1200 \mathrm{~cm}^{-1}$ region, characteristic for the Si-O-Si asymmetric stretching in the bulk silica systems, led the authors to the conclusion that the film does not exhibit Si-O-Si bonds. Therefore, the authors have proposed a model consisting of a layer of isolated $\left[\mathrm{SiO}_{4}\right]$ clusters arranged in a $\mathrm{c}(2 \times 2)$ structure on the $\mathrm{Mo}(112)$ surface with all oxygen atoms bonding to the Mo substrate as shown in Fig. 1a. This model is hereafter referred to as a "cluster model".

The interest in the structure of ultra-thin films was reinforced after Weissenrieder et al. [32], modifying the preparation procedure, reported a scanning tunneling microscopy (STM) study of the silica films with atomic resolution. The STM images revealed a honeycomb-like surface on the extended flat terraces separated by the monoatomic steps of the Mo substrate underneath (Fig. 2a). In addition, well-resolved images of antiphase domain boundaries (APDB) running along the [110] direction as predicted by LEED studies [18] were obtained as indicated in Fig. 2a. These results, combined with IRAS and XPS data (discussed below) obtained on the same samples, suggested that the film is indeed of a monolayer thickness but consists of $\left[\mathrm{SiO}_{4}\right]$ tetrahedra with one apex $\mathrm{O}$ atom pointing to the Mo surface whereas the surface is formed by a fully saturated $\mathrm{O}$ layer arranged in honeycomb structure through sharing the corner O-atoms (see Fig. 1c). (Note, that this structure was suggested but rejected by 
Chen et al [28].) This model, which is henceforth referred to as the "2D-network model", was further corroborated by density functional theory (DFT) calculations [32,36]. Simultaneously, a similar 2D-network model of the film was independently proposed by Pacchioni and co-workers [33] who also discarded the cluster model for the silica film.

However, very recently, Chen and Goodman [43] reported an STM study, which in their opinion favors the cluster model, although slightly modified as shown in Fig. $1 \mathrm{~b}$. In addition, they discussed issues in interpreting vibrational spectra of thin films and referred to the cluster calculations of Yakovkin [34].

In this paper, we have attempted to critically review all the experimental and theoretical studies on thin crystalline silica films reported to date. In addition, we provide new photoelectron spectroscopy results using synchrotron radiation and also report an UPS study, which strongly support the 2D-network model of the monolayer silica film. This review also highlights the crucial role of theory in the determination of atomic structure of the silica films and in interpretation of their vibrational spectra.

The paper is organized as follows. We first describe the details of the silica film preparation, which turn out to be critical for the structures of the films grown. Then we summarize the key observations of experimental and theoretical studies on this system reported in the literature and discuss how these results agree or disagree with the models suggested. The paper ends with a summary and concluding remarks.

\section{Film preparation}

There are different preparations of the silica film on $\operatorname{Mo}(112)$ reported in the literature. Originally, Schroeder et al. [15] used four cycles of Si deposition at $300 \mathrm{~K}$ and oxidation at $800 \mathrm{~K}$ in $5 \times 10^{-6}$ mbar $\mathrm{O}_{2}$. Subsequently, the film was annealed stepwise up to $1100-1250 \mathrm{~K}$ in $10^{-5}$ mbar of $\mathrm{O}_{2}$ until the films exhibited a $\mathrm{c}(2 \times 2)-\mathrm{Mo}(112)$ structure by LEED.

Chen et al. [28] have modified the recipe by first exposing a clean $\operatorname{Mo}(112)$ surface to $5 \times 10^{-8}$ mbar $\mathrm{O}_{2}$ at $850 \mathrm{~K}$, which produces the $\mathrm{p}(2 \times 3) \mathrm{O}-\mathrm{Mo}(112)$ surface. Submonolayer amounts of Si were then deposited onto this surface at $300 \mathrm{~K}$, followed by annealing at $800 \mathrm{~K}$ in $10^{-7} \mathrm{mbar}_{2}$ for $5 \mathrm{~min}$ and increasing the temperature to $1200 \mathrm{~K}$ 
for additional $5 \mathrm{~min}$. These steps were repeated several times until a constant $\mathrm{Si} / \mathrm{Mo}$ Auger ratio was obtained. Another preparation basically omitted the intermediate annealing step, and only the completed film was subjected to annealing at temperatures that range from 1000 to $1200 \mathrm{~K}$. In addition to these "multistep" preparations, the same group later used a single step preparation by deposition of 1.5-2 monolayer (ML) of Si onto the $\mathrm{p}(2 \times 3) \mathrm{O}-\mathrm{Mo}(112)$ surface at $300 \mathrm{~K}$ followed by oxidation in $10^{-7} \mathrm{mbar}_{2}$ at 800 $\mathrm{K}$ for $10 \mathrm{~min}$ and annealing at $1050-1250 \mathrm{~K}$ using the same oxygen pressures.

Finally, our own group prepared the films by exposing the clean $\operatorname{Mo}(112)$ surface to $5 \times 10^{-8} \mathrm{mbar}_{2}$ at $900 \mathrm{~K}$ (resulting in $\mathrm{p}(2 \times 3) \mathrm{O}-\mathrm{Mo}(112)$ surface) and subsequent deposition of approximately 1.2 $\mathrm{ML}$ of $\mathrm{Si}$ at the same oxygen pressure and temperature. The films were then annealed in UHV at ca. $1250 \mathrm{~K}$. The annealing temperature has turned out to be critical. Based on the STM studies we have found that overheating results in partial sublimation of the film, while low temperature annealing results in the presence of ill-defined silica particles on top of the ordered film. The preparation involving final annealing in UHV produces the film, which is referred to as "O-poor". In contrast, high temperature annealing in $\sim 10^{-6}$ mbar $\mathrm{O}_{2}$ during the preparation or, alternatively, annealing of the "O-poor" films in oxygen results in the "O-rich" films, in which additional oxygen atoms underneath the film are adsorbed onto the Mo substrate [37] (see Fig. 1d). Therefore, the preparations reported by Schroeder et al. [15] and Chen et al. [28], which include annealing in oxygen ambient, must have resulted in the "Orich" films in our notation.

\section{Low energy electron diffraction (LEED)}

All well-ordered silica films prepared by different recipes showed a $\mathrm{c}(2 \times 2)$ Mo(112) diffraction pattern. Chen et al. [28] depicted several possible models which may explain the $c(2 \times 2)$ structure. In principle, both cluster and 2D-network models exhibit this symmetry. Note, however, that a spot-profile analysis of the diffraction patterns performed by Schroeder et al. [18] revealed a structure containing two domains with antiphase domain boundaries (APDB) between the domains propagating along the [110] direction. 
The presence of additional oxygen atoms observed in the "O-rich" films does not change the symmetry of the film and cannot be discerned by conventional LEED. To the best of our knowledge, no dynamic (I/V) LEED studies are reported to date.

\section{Scanning Tunneling Microscopy (STM)}

Determination of the atomic structure of the monolayer silica film using electron and vibrational spectroscopies, which will be discussed in the following sections, cannot be done without precise control of the morphology of the silica films under study, even though LEED may show a sharp diffraction pattern. In particular, the samples may contain small silica particles and clusters, whose properties may strongly influence the spectroscopic results. The systematic STM studies performed in [36] revealed that atomically flat films can be produced only after annealing at temperatures above $1200 \mathrm{~K}$. It should be also emphasized that STM images of the thin oxide films very often cannot be interpreted straightforwardly and therefore other supporting information is necessary to prove a proposed structure model (see, for example, $[9,12])$.

Figures $2 \mathrm{~b}$ and $2 \mathrm{~d}$ show high-resolution STM images obtained on the "O-poor" films. A honeycomb-like structure resolved in Fig. 2d was explained within the 2Dnetwork model by assigning the protrusions in this image to the oxygen atoms in the topmost O-layer. Indeed, the image simulation performed on the basis of the calculated density of states for this structure showed a good match with the experiment (Fig. 2). Furthermore, at certain tunneling parameters the Si atoms could be imaged (Fig $2 \mathrm{~b}$ ) and, again, this fact has been proven by comparing the experimental and simulated STM images $[32,36]$.

Figure 2 also shows the line defects running along the Mo[110]direction, as previously observed by LEED [18], which appear as alternating 8-members and 4members rings. These straight-line defects can be easily explained within the $2 \mathrm{D}$-network model by a half-lattice shift in the [ $\overline{1} \overline{1} 1]$ direction between two domains as shown in Fig. 2e. In contrast, the cluster model will result in the APDB running in the $[\overline{3} 11]$ direction, which is inconsistent with the LEED results. 
Interestingly, high-resolution images also showed modulation in corrugation amplitude on a large scale (see Fig. 2a). In a first approximation, this can be explained as a result of the small lattice mismatch between the oxide film and the substrate, which often leads to the periodic Moire structure as observed for monolayer films of $\mathrm{FeO} / \mathrm{Pt}$ [46], $\mathrm{TiO}_{\mathrm{x}} / \mathrm{Pt}$ [47], $\mathrm{Nb}_{2} \mathrm{O}_{5} / \mathrm{Cu}_{3} \mathrm{Au}$ [48], etc. However, in the case of silica, one should take into account a high flexibility of the Si-O-Si bonds that may explain the "random wave" modulation observed. Another explanation is based on the presence of small amounts of the sub-surface impurities in the Mo crystal.

At sub-monolayer coverage, high-resolution STM studies revealed formation of $0.5 \mathrm{~nm}$-wide silica stripes along the $[\overline{1} \overline{1} 1]$ direction. These stripes, which exhibit a $(1 \times 3)$ periodicity, cannot be assigned to the oxygen reconstructed $\operatorname{Mo}(112)$ surface which showed completely different STM images on the Si-free samples prepared under the same conditions in the blank experiments. The stripes coalesce and form islands with the same structure as the dense silica film as shown in Fig. 3a. Based on the registry analysis of the STM images and complementary XPS, IRAS, and DFT results [40], these stripes were explained by corner-sharing pairing of the $\left[\mathrm{SiO}_{4}\right]$ tetrahedra adsorbed on the $(1 \times 3)$ reconstructed Mo surface as shown in Fig. 3b. In particular, DFT calculations have nicely explained a strong $\left(\sim 100 \mathrm{~cm}^{-1}\right)$ blue-shift of one of the vibrational bands observed in the IRA-spectra of the silica stripes (see below). In contrast, these stripes can hardly be explained within the cluster model since the clusters pairing would result in unreasonably short distance between $\mathrm{O}$ atoms of two adjacent $\left[\mathrm{SiO}_{4}\right]$ clusters, and therefore will be thermodynamically unstable.

Very recently, Chen and Goodman [43] have reported STM images which, in their opinion, favor the cluster model. Note that the images presented in their paper appear to suffer from tip effects (the authors obtained different images at the same tunneling parameters, compare (a) and top of (j) in Fig. 3 in ref. [43]). In attempts to rationalize the key STM image (reproduced in Fig. 2c), the authors had to modify the original cluster model as shown in Figs. 1a,b. However, no STM simulations have been applied to prove the structure. Meanwhile, the image simulation, performed for the cluster model by Todorova et al. [36] showed that isolated $\left[\mathrm{SiO}_{4}\right]$ clusters must appear in STM as large single spots (see Fig. 2g), at least at the tunneling parameters applied in the 
experiment, which does not fit the experimental observations. It should be emphasized that the films prepared by Chen et al. $[28,43]$ by annealing in oxygen environment must be "O-rich" in nature and therefore the corresponding STM images can be strongly influenced by the presence of the additional oxygen atoms chemisorbed on Mo. All in all, the analysis of STM images alone cannot determine the structure of the silica film in a definitive manner. The images observed must be complemented with information gathered from other techniques [43].

\section{X-ray photoelectron spectroscopy (XPS)}

Photoelectron spectroscopy (PES) of the core levels, when applied to the oxide surfaces, is a principal tool for determining the oxidation state of the metal cations and average oxide stoichiometry. PES can resolve different oxygen and/or cation species present on oxide surfaces. In addition, analysis of angular resolved photoelectron spectra may provide useful information on the depth distribution of the different species.

In all studies reported the $\mathrm{Si} 2 p$ region showed only a single peak centered at $\sim 103$ $\mathrm{eV}$, which was straightforwardly assigned to the tetrahedrally coordinated $\mathrm{Si}$ atoms in oxidation state $4+[49,50]$. Regarding the oxygen states, at least two different $\mathrm{O}$ species were observed $[15,29]$ with binding energies (BE) of the O1s level at $530.3(530.7)$ and $532(532.2) \mathrm{eV}$, i.e. separated by approximately $1.5 \mathrm{eV}$. The high BE values fall into the region reported for the bulk silica or "thick" silica films $[49,50]$. Schroeder et al. [18] assigned these signals to $\mathrm{O}$ species at the silica/Mo interface and in top layer of the silica film, respectively. Based on the literature data available for alkaline and transition metal oxide surfaces, Min et al. [29] concluded that the $530.3 \mathrm{eV}$ feature must be assigned to the interface (Si-O-Mo) bonds, and the $532.0 \mathrm{eV}$ signal to oxygen in the $\mathrm{Si}-\mathrm{O}-\mathrm{Si}$ bonds. Note that this assignment contradicts the cluster model suggested by the same group [28], where only Si-O-Mo bonds are present.

Further XPS studies have verified formation of two different $\mathrm{O}$ species in the "Opoor" silica films [32,36]. Two overlapping signals centered at $\sim 532.5$ and $\sim 531.2 \mathrm{eV}$ were observed. The atomic ratio $(3: 2)$ estimated from spectral deconvolution agrees well with the ratio between surface $(\mathrm{O} 1+\mathrm{O} 2$, Fig. 1c) and interface $(\mathrm{O} 3)$ oxygen atoms within 
the 2D-network model. Note that the STM study of these films confirms the absence of silica particles which may influence this ratio. The theoretical calculations of the core level shift for the $2 \mathrm{D}$-model revealed the value of $1.3 \mathrm{eV}$, in a perfect agreement with experimental observations. On the contrary, calculations performed for the DFToptimized $\left[\mathrm{SiO}_{4}\right]$ cluster model, shown in Fig. 2f, predicted three states within $0.8 \mathrm{eV}$ range and therefore would result in a single and broad O1s peak. This is in clear disagreement with experimental observations.

In order to perform a quantitative analysis of the absolute amounts of oxygen in the "O-poor" and "O-rich" silica films, in the present work we have calibrated the spectrometer on the well-known $(2 \times 1) \mathrm{O}-\mathrm{Ru}(0001)$ surface right before the measurements on the silica. The values measured (24 \pm 4 and $28 \pm 5 \mathrm{at} / \mathrm{nm}^{2}$, respectively) are consistent with the values predicted by the 2D-network model $\left(=20.6\right.$ and $\left.28.8 \mathrm{at} / \mathrm{nm}^{2}\right)$ and far above the amount predicted by the cluster model $\left(=16.5 \mathrm{at} / \mathrm{nm}^{2}\right)$.

The presence of "extra" oxygen atoms in the "O-rich" films manifests itself in the feature at $530.6 \mathrm{eV}$ [37], i.e., close to that observed on the $\mathrm{O} / \mathrm{Mo}(112)$ surface under the same conditions (see also [51]). This feature overlaps with the signal of interface (Si-OMo) oxygen at $531.2 \mathrm{eV}$. In addition, a high BE shoulder was observed in the Mo3d region [37], which was explained by a larger charge transfer through the O-Mo bond as compared to the Si-O-Mo bonds.

To increase the spectral resolution, we have employed synchrotron facilities at BESSY II, Berlin (beam-line UE52-PGM1). The O1s region of the "O-poor" silica film is shown in Fig. 4, where two oxygen species at $532.5 \mathrm{eV}$ and $531.2 \mathrm{eV}$ are clearly resolved. When measured at grazing electron emission $\left(80^{\circ}\right.$ with respect to the surface normal), the low BE signal has virtually vanished, indicating that these species are located below the layer consisting of $\mathrm{O}$ species with the higher BE. This finding fully agrees with the previous assignments of the $531.2 \mathrm{eV}$ peak to the interface (Si-O-Mo) oxygen atoms and $532.5 \mathrm{eV}$ peak to the topmost O layer within the Si-O-Si network $[32,36]$. Since these two peaks are well separated, the integral intensity ratio can be precisely measured and is found to be $3: 2$ at normal emission, as predicted by the 2Dnetwork model as the ratio between the $(\mathrm{O} 1+\mathrm{O} 2)$ and $\mathrm{O} 3$ oxygen atoms. In addition, the 532.5 peak is broader than the 531.2 peak since it consists of two slightly different 
oxygen species, $\mathrm{O} 1$ and $\mathrm{O} 2$. In contrast, any of the cluster models presented in Figs. 1a,b must result in (i) close $\mathrm{BEs}$ for the $\mathrm{O}$ atoms in the $\left[\mathrm{SiO}_{4}\right]$ cluster and (ii) a 1:1 ratio for the two Si-O-Mo linkages.

Figure 4 shows the Mo3d region, where only one partially oxidized Mo species at $228.2 \mathrm{eV}$ is detected beyond the metallic state at $228 \mathrm{eV}$. (Note that the spectrometer resolution was sufficient to resolve up to four different $\mathrm{O}$ induced Mo states on the oxygen reconstructed $\mathrm{Mo}(112)$ surface). At grazing emission, the oxidized state slightly gain intensity as compared to the metallic state due to its surface nature. Again, these results are consistent with the 2D-network model, where only one Si-O-Mo linkage exists, but disagree with the cluster model, where there must be two different Si-O-Mo species. One may argue, that the two Si-O-Mo linkages are identical and therefore cannot be resolved for the Mo3d level. However, this automatically implies the presence of only one $\mathrm{O} 1 \mathrm{~s}$ state and hence contradicts the experimental results for the O1s core level, where two O species are clearly observed.

\section{Ultraviolet Photoelectron Spectroscopy (UPS).}

Schroeder et al. [18] observed three main peaks in the UP-spectra of the silica films between 4 and $12 \mathrm{eV}$ as shown in Fig. 5a. Based on the literature data, the signal between 10 and $12 \mathrm{eV}$ was attributed to the hybridized $\mathrm{O} 2 p$-Si3s,3p bonding states, and the two peaks between 4 and $9 \mathrm{eV}$ - to nonbonding $\mathrm{O} 2 p$ derived valence band.

Using UPS and metastable impact electron spectroscopy (MIES) data, Wendt et al. [35] assigned a band centered at around $11.5 \mathrm{eV}$, observed on the "thick" silica films, to the Si-O-Si bonds. Consequently, a band at $10.4 \mathrm{eV}$, that emerges for the monolayer films (see Fig. 5b), was attributed to the Si-O-Mo bond, where the interface $\mathrm{O}$ atoms likely accumulate electron density from the Mo substrate.

Fig. 5c shows the UP-spectrum of the "O-poor" film prepared in our laboratory. It is noteworthy that very narrow bands confirm a high crystallinity of the silica film, which does not have any silica particles on the surface as evidenced by STM. The peak assignments can be done based on theoretical and experimental studies of different silica polymorphs $([52,53]$ and references therein). 
The bands between 5 and $8 \mathrm{eV}$ can be straightforwardly assigned to the $\mathrm{O} 2 \mathrm{p}$ nonbonding states. Based on symmetry considerations, splitting of nonbonding orbitals is explained in terms of nonbonding $p_{x}$ and $p_{y}$ orbitals, which lie perpendicular and parallel to a plane determined by the Si-O-Si bond [52]. Such a splitting is expected in silica compounds with the Si-O-Si bond angle deviated from $180^{\circ}$. Wendt et al. [35] considered this splitting to be unlikely in the crystalline silica film and assigned it to isolated $\left[\mathrm{SiO}_{4}\right]$ tetrahedra, where $\mathrm{O} 2 \mathrm{p}$ non-bonding orbitals can be bent $90^{\circ}$ away from each other. However, the 2D-network model of the film shown in Fig. 1c contains two Si-O-Si linkages with bond angles of $133^{\circ}$ and $163^{\circ}$ [36], which therefore may explain the observed band splitting, too.

In the bonding region of the UP-spectrum (c) in Fig. 5, the two signals at 11.4 and $10.6 \mathrm{eV}$ (as a prominent shoulder) are associated with Si-O-Si and Si-O-Mo bonds, respectively, i.e., in agreement with the assignment of Wendt et al. [35]. The absence of the $11.5 \mathrm{eV}$ feature in the spectra previously reported by Schroeder et al [18] (and also by Wendt et al. [35] for the film grown on mildly oxidized Mo(112)) can be explained by the fact that those films, according to the preparations used, were "O-rich" in nature. Indeed, the O-rich films contain more $\mathrm{O}$ atoms bonded to the Mo substrate in different adsorption sites depending on the oxygen pressure ([37], see Fig. 1d). Therefore, the signal at $\sim 10.5 \mathrm{eV}$ for the "O-rich" films may prevail over the band at $\sim 11.5 \mathrm{eV}$ and gets broader. In addition, based on the lack of spectral resolution in the $\mathrm{O} 2 p$ nonbonding region (between 4 and $9 \mathrm{eV}$ ) in the spectra (a) and (b) shown in Fig. 5, the films were likely not well-ordered (no STM data available for the films measured), which again might led to signal broadening such that the feature at $\sim 11.5 \mathrm{eV}$ can hardly be resolved on these "O-rich" films.

In summary, both core level and valence band photoelectron spectroscopy results show the existence of both Si-O-Si and Si-O-Mo bonds in the monolayer film, which are consistent with the 2D-network model and inconsistent with the cluster model.

\section{Near edge X-ray absorption fine structure (NEXAFS) spectroscopy}


Near edge X-ray absorption fine structure (NEXAFS) spectroscopy, a technique that probes electronic transitions from core levels into unoccupied states, is a powerful technique for determining local atomic geometry, bond angles and electronic structure of the unoccupied states $[54,55]$. Figure 6 a shows O K-edge NEXAFS spectrum measured with synchrotron radiation at BESSY II. According to the dipole selection rule, only transitions from an initial $s$ state to a final $p$ state are allowed, therefore the unoccupied states with $\mathrm{O}$ p-like character are probed in these spectra $[54,55]$.

The most prominent feature in the measured NEXAFS spectra is the strong absorption in the region between $536 \mathrm{eV}$ and $547 \mathrm{eV}$. For comparison, for oxidized Mo(112) surfaces we observed strong absorption into the hybridized Mo3d - O2p orbitals at $530-535 \mathrm{eV}$ (not shown here) in agreement with previous studies on molybdenum oxide compounds $[56,57]$. In the "O-poor" $2 \mathrm{D}$-network model only $40 \%$ of the $\mathrm{O}$ atoms are involved in bonds with the Mo substrate, while all the $\mathrm{O}$ atoms bind directly to the Mo surface in the cluster model. As a consequence, the contribution of the Mo-O bonds to the spectra is expected to be significantly larger for the cluster model than the 2Dnetwork model. In fact, Fig. 6 shows only minor absorption in the region below $535 \mathrm{eV}$. Moreover, our spectra have turned out to be very similar to the spectra reported in the literature for $\alpha$-quartz [58-60]. Also, the spectra revealed resemblance to the calculated local density of states for $\alpha$ - and $\beta$-cristobalite [60]. All of these three silica compounds are constructed by corner sharing $\left[\mathrm{SiO}_{4}\right]$-tetrahedra, i.e. like in the 2D-network model proposed. In contrast, the NEXAFS spectra from rutile stishovite with corner and edge sharing $\left[\mathrm{SiO}_{6}\right]$-octahedra are significantly different $[61,62]$. It is the difference in local atomic geometry that gives rise to different crystal field splitting of the relevant orbitals. The close similarities observed between the spectra for the silica film and the reference silica compounds clearly indicate the presence of Si-O-Si bonds in the film as a result of a corner sharing geometry.

Finally, Fig. $6 \mathrm{~b}$ shows calculated projected O $2 \mathrm{p}$ local density of states (DOS) above Fermi level, probed by NEXAFS, for the cluster and 2D-network models. It is clear that the states predicted for the cluster model cannot reproduce the experimental data since the absorption signals are expected in the region below $536 \mathrm{eV}$, whereas the 2D-network model accurately predicts not only the band position but also the overall 
shape of the measured spectrum. Therefore, in addition to photoelectron spectroscopy results, NEXAFS spectroscopy provides more evidence for the 2D-network model.

\section{Stability of Different Models}

The first attempt to employ theoretical calculations using DFT to determine the structure of the crystalline $\mathrm{SiO}_{2} / \mathrm{Mo}(112)$ film was made by Ricci and Pacchioni [27]. The authors investigated the stability and adhesion energies of thin films of known silica modifications on $\mathrm{Mo}(112)$. They concluded that the $\mathrm{SiO}_{2} / \mathrm{Mo}(112)$ film most probably consists of three $\mathrm{SiO}_{2}$ layers of the $\beta$-cristobalite-like structure because it provides the strongest adhesion to the $\operatorname{Mo}(112)$ surface and the smallest strain in the epilayer. However, the results of calculations on the $\beta$-cristobalite model do not show satisfactory agreement with experimental results for the $\mathrm{SiO}_{2} / \mathrm{Mo}(112)$ film $[27,36]$.

Our recent DFT calculations [36,37] and independent calculations performed by Giordano et al. [33] reached the conclusion that the most stable form of the $\mathrm{SiO}_{2} / \mathrm{Mo}(112)$ film is the $2 \mathrm{D}$ network model. Here, we present a summary of the theoretical stability calculations on all four atomic structure models proposed for the crystalline $\mathrm{SiO}_{2} / \mathrm{Mo}(112)$ film, the $\beta$-cristobalite [27], isolated $\left[\mathrm{SiO}_{4}\right]$ cluster [28], and 2D-netwok "O-poor" and "O-rich" models [36,37]. For comparing the stabilities of the different structure models we consider their hypothetical formation from a clean $\mathrm{Mo}(112)$ surface, bulk $\alpha$-quartz, and oxygen:

$$
\mathrm{Mo}(112)+m\left(\mathrm{SiO}_{2}\right)_{\alpha \text {-quartz }}+n \frac{1}{2} \mathrm{O}_{2} \rightarrow\left(\mathrm{SiO}_{2}\right)_{m} \cdot n \mathrm{O} / \mathrm{Mo}(112),
$$

where $m$ and $n$ are the number of $\mathrm{SiO}_{2}$ units and oxygen excess in a surface unit cell, respectively. (Note that in contrast to the statement made by Chen et al. [45] this definition of $\Delta E_{\text {form }}$ does not involve any extra oxygen atoms bonded to the clean $\operatorname{Mo}(112)$ substrate to maintain the same atom number on both sides of the Eq. 1).

The formation energy, $\Delta E_{\text {form }}$, is obtained from the total energies of the different systems in Eq. 1 which are calculated by DFT. For the solid systems, periodic boundary conditions are applied, and surfaces are treated as periodic slabs. The input of the DFT calculations is only the type and number of atoms in the unit cell. The positions of the 
atoms are obtained by energy minimization. The calculated $\Delta E_{\text {form }}$ values are $-9.1 \mathrm{eV}$ for the $\beta$-cristobalite model $(m=10, n=3),-11.5 \mathrm{eV}$ for the $\left[\mathrm{SiO}_{4}\right]$ cluster model $(m=2, n=$ $4)$, as well as $-10.8 \mathrm{eV}$ and $-24.2 \mathrm{eV}$ for the "O-poor" $(m=4, n=2)$ and "O-rich" $(m=4$, $n=6) 2 \mathrm{D}$-network models, respectively. Although it is clear that the "O-rich" $2 \mathrm{D}$ network model is the most stable structure in terms of $\Delta E_{\text {form }}$ we have to compare the stability of the different models at given experimental conditions such as partial oxygen pressure $\left(p_{\mathrm{O}_{2}}\right)$ and temperature $(\mathrm{T})$. This comparison can be made using the free energy change $(\Delta \gamma)$ of reaction (1) per surface area $(S)[63]$ :

$$
\Delta \gamma(T, p)=\frac{1}{S}\left[\Delta E_{\text {form }}-n \Delta \mu_{\mathrm{O}}(T, p)\right]
$$

with $\Delta \mu_{\mathrm{O}}(T, p)=\mu_{\mathrm{O}}-1 / 2 E_{\mathrm{O}_{2}}$, where $\mu_{\mathrm{O}}$ is the oxygen chemical potential and $E_{\mathrm{O}_{2}}$ is the energy of $\mathrm{O}_{2}$ molecule. The relative oxygen chemical potential $\Delta \mu_{\mathrm{O}}(T, p)$ is related to temperature and partial oxygen pressure by assuming that the surface is in thermodynamic equilibrium with the gas phase $\mathrm{O}_{2}$ [63]. The stability plot of $\Delta \gamma(T, p)$ for the selected models is presented in Fig. 7, which clearly shows that the 2D-network models are the most stable systems at any experimentally relevant conditions. Note, that the existence of the "O-rich" phase of the 2D film has not been explicitly considered by Chen and Goodman in their recent publications [43,45], particularly concerning qualitative analysis of the stability of different silica film models.

\section{Vibrational spectroscopy: Experiment vs Theory}

Vibrational spectroscopy is a useful tool for characterization of thin oxide films (see examples in [3,6,9,12]). Schroeder et al. [18] reported IRA spectra at the different stages of the $\mathrm{SiO}_{2} / \mathrm{Mo}(112)$ film preparation. The main signal at $1048 \mathrm{~cm}^{-1}$ with a full width at half maximum (FWHM) of $\sim 40 \mathrm{~cm}^{-1}$ (Fig. 8), observed for the ordered films, as judged by LEED, was assigned to asymmetric Si-O-Si stretching vibrations. A strong frequency shift from $1150-1200 \mathrm{~cm}^{-1}$ (typically observed for this vibration mode in the bulk silica) was attributed to the presence of the Si-O-Mo bonds. In addition, two weak bands at 795 and $675 \mathrm{~cm}^{-1}$ were observed, and the latter vibration was assigned to the O- 
Mo vibrational modes at the interface. Ricci and Pacchioni [27] also suggested that the $795 \mathrm{~cm}^{-1}$ feature is associated with the presence of two-membered silica rings on the surface of the $\beta$-cristobalite film model used in their calculations.

Based on the HREELS study of the silica films and the literature data for various Si and Mo oxide compounds, Chen et al. [28] have suggested that the band at $1048 \mathrm{~cm}^{-1}$ (shown in the inset in Fig. 8) must be assigned to the Si-O-Mo stretching vibration. The absence of the band at $\sim 1176 \mathrm{~cm}^{-1}$, observed for the thicker silica films and assigned to asymmetric Si-O-Si stretching, has been interpreted to mean that the ultra-thin film does not exhibit Si-O-Si bonds at all and consists of exclusively $\left[\mathrm{SiO}_{4}\right]$ clusters with the Si-OMo linkages only.

Recently, Yakovkin [34] reported calculation of vibrational spectrum for the $\left[\mathrm{SiO}_{4}\right]$ cluster model using only a small molecular model of the surface containing just one $\left[\mathrm{SiO}_{4}\right]$ unit. Such a model cannot adequately describe the Mo conduction band electrons and the coupling between vibrational modes of the silica layer and the substrate. Additionally, the Hartree-Fock method applied by the author along with a minimal STO$3 \mathrm{G}$ basis set is well known to be particularly inaccurate for description of transition metal containing compounds. Therefore, in our opinion the reported agreement between the calculated Si-O stretching frequency at $1044 \mathrm{~cm}^{-1}$ and experimental value at $1048 \mathrm{~cm}^{-1}$ is purely accidental.

On the atomically flat "O-poor" films, we have observed a very strong and sharp IRAS peak at $1059 \mathrm{~cm}^{-1}\left(\mathrm{FWHM} \sim 12 \mathrm{~cm}^{-1}\right)$. In agreement with Chen et al. [28], we have also assigned this signal to the asymmetric Si-O-Mo stretching. The main signal gets broader and shifts to lower energies by $\sim 10 \mathrm{~cm}^{-1}$ in the films prepared by annealing in oxygen (i.e. "O-rich" film) [37]. The broad band centered at $\sim 1200 \mathrm{~cm}^{-1}$ (not shown here) emerges only on the films containing silica particles [36], which is therefore assigned to asymmetric Si-O-Si stretching in the bulk silica. However, the absence of the absorption bands characteristic for the $\mathrm{Si}-\mathrm{O}-\mathrm{Si}$ vibrations in the monolayer film does not straightforwardly mean the absence of the $\mathrm{Si}-\mathrm{O}-\mathrm{Si}$ bonds as postulated by Chen et al. [28]. In fact, this effect can be rationalized on the basis of the selection rules applied to the refractory metal surfaces. 
The selection rule (which holds true for the thin films as well) states that only vibrational modes with a component of the dynamic dipole moment perpendicular to the metal surface will contribute to the IR absorption (see e.g. ref. [64]). These vibrational modes are, in general, only the totally symmetrical modes of the film $[65,66]$.

To apply the selection rules to the 2D-network model, we note first that the surface unit cell belongs to the two-dimensional $\mathrm{cmm}$ space symmetry group which contains eight symmetry elements as shown in Fig. 9a and listed in Table 1. Moreover, since the unit cell contains four $\mathrm{Si}$ and ten $\mathrm{O}$ atoms, a straightforward analysis shows that there will be six normal modes involving coupled asymmetric $\mathrm{Si}-\mathrm{O}-\mathrm{Si}$ stretching vibrations, six modes involving coupled symmetric Si-O-Si stretching vibrations, and four modes involving coupled asymmetric Si-O-Mo stretching vibrations. Figs. 8-10 depict the vibrational modes of the 2D-network model along with corresponding calculated frequencies, and Table 1 shows their transformation properties with respect to symmetry elements of the $\mathrm{cmm}$ space group. It is important to note that only the frequency values are obtained from the DFT calculations, the normal modes themselves can be deduced from the composition of the unit cell and its symmetry alone. These vibrational modes comprise all vibrations calculated for the 2D-network model in the range of $1200-650 \mathrm{~cm}^{-1}$ as measured in the IRAS experiments.

The six asymmetric Si-O-Si stretching vibrations shown in Fig. 9b are IRAS inactive, because there is no dynamic dipole moment component perpendicular to the surface associated with any of the couplings of the asymmetric Si-O-Si stretching modes. The only vibrations that can result in the IR absorption are the asymmetric Si-O-Mo stretching and symmetric Si-O-Si stretching vibrations. Table 1 demonstrates that there are only three vibrational modes with frequencies at 1061, 779 and $672 \mathrm{~cm}^{-1}$ that are symmetric with respect to all symmetry operations of the $\mathrm{cmm}$ symmetry group. These are in-phase couplings of the asymmetric Si-O-Mo modes as well as out-of-phase and inphase couplings of the symmetric Si-O-Si stretching vibrations, respectively (the latter two can be alternatively interpreted as $\mathrm{Si}-\mathrm{O}-\mathrm{Si}$ symmetric stretching coupled with $\mathrm{Si}-\mathrm{O}-\mathrm{Si}$ bending modes). These are precisely the three modes observed in the IRAS experiments as shown in Fig. 8. All other vibrations in the range of $1200-650 \mathrm{~cm}^{-1}$ are IR inactive because of the symmetry selection rule. 
The symmetry considerations as presented above can be applied both to "O-poor" and "O-rich" 2D films since both structures exhibit the same symmetry. In case of the "O-rich" film, the calculated main Si-O-Mo stretching mode exhibits a red-shift by about $15 \mathrm{~cm}^{-1}$, as indeed observed in experiment (see Fig. 8). Similar analysis has been applied to the silica stripes formed at sub-monolayer silica coverage (see Fig. 3) [40]. The major difference between stripes and film is observed for the mode at $879 \mathrm{~cm}^{-1}$, which is shifted by about $100 \mathrm{~cm}^{-1}$ towards higher wavenumbers as compared to the 2D-films (see Fig. 8).

Symmetry analysis of the vibrational modes for the $\left[\mathrm{SiO}_{4}\right]$ isolated tetrahedra adsorbed in the trenches of the Mo(112) surface as shown in Fig. 1a is very simple. The site symmetry of the adsorbed $\left[\mathrm{SiO}_{4}\right]$ tetrahedron is $\mathrm{C}_{2 \mathrm{v}}$ and it follows from an elementary symmetry analysis (see, for example, textbook [67]) that only two symmetric and two asymmetric combinations of the Si-O stretching vibrations exist for this model. Only the two symmetric combinations are completely symmetrical with respect to all symmetry operations of the $\mathrm{C}_{2 \mathrm{v}}$ symmetry group and therefore can be observed by IRAS. This is in agreement with our DFT calculations [36], which showed that for the cluster model basically only two symmetric O-Si-O vibrational modes at 706 and $532 \mathrm{~cm}^{-1}$ are IRAS active. This is in contrast to the claim $[43,45]$ that the asymmetrical Si-O vibrations of the $\left[\mathrm{SiO}_{4}\right]$ tetrahedron should be always IR active.

In a dipole scattering regime HREELS obeys the same selection rule that applies to infrared spectroscopy. Only the completely symmetrical vibrational modes are allowed when the interaction is between the electrical field of the incoming electron and the vibrational dipole moment of the film. In this long-range interaction the reflected electrons occur close to the specular direction [65,68]. As a result, the HREELS experiments performed for the monolayer silica film showed the same three vibrational modes in the range of $600-1200 \mathrm{~cm}^{-1}$ as seen by IRAS [28].

However, in the inelastic scattering regime of HREELS also the bands associated with vibrations parallel to the metal surface can be observed. These impact features show much weaker intensity than the dipole scattered ones and are best observed at offspecular angles. Additionally, the inelastic scattering features obey the following symmetry selection rules. First, if the plane of incidence of the electron beam coincides with a plane of symmetry, then a vibration that is antisymmetrical with respect to that 
plane is forbidden in both specular and non-specular directions. Such modes could be observed only in off-specular directions in the plane perpendicular to the incident plane [65]. Second, if a vibrational mode is antisymmetric with respect to a two-fold axis of symmetry or plane of symmetry perpendicular to the surface and to the plane of incidence of the electrons, then the mode is again forbidden in the specular direction [65]. It is evident from Table 1 that all modes, except the totally symmetric modes at 1061, 779, and $672 \mathrm{~cm}^{-1}$, are antisymmetric with respect to at least one two-fold symmetry axis and one symmetry plane. Therefore, it is not surprising that the intensity of the vibrations is extremely low and renders their detection impossible even for off-specular HREELS measurements.

There is also a misleading use of terminology concerning transverse (TO) and longitudinal (LO) polar optical vibrational modes for the silica film $[43,45]$. The terminology TO and LO modes originates from a vibrational analysis of an infinite cubic crystal with two atoms in a unit cell [69-72]. In such crystals plane TO modes occur at a frequency $\omega_{\text {TO }}$ such that the imaginary part of the complex dielectric function $\left(\varepsilon^{*}\right)$ has a maximum value. The LO modes occur at a frequency $\omega_{\text {LO }}$ where $\varepsilon^{*}$ has a minimum absolute value. Additionally, in the infinite cubic crystal the LO phonons induce a longrange electric field in the crystal which opposes the motion of the ions thus increasing the $\omega_{\text {LO }}$ frequency as compared to $\omega_{\text {TO }}[71,72]$. In analogy with the infinite crystal, the TO and LO terminology has also been used for a crystalline film $[69,70]$. The film has two normal modes of polarized lattice vibrations with the wavelength much greater than the film thickness. In one mode the vibrations are parallel to the film surface and the frequency is $\omega_{\text {TO }}$. The term TO is used because the vibrations parallel to the film surface produce no electric field, so they occur when the dielectric function has a very large absolute value. In the other mode, the vibrations are normal to the film surface and the frequency is $\omega_{\mathrm{LO}}$. Again, the term LO is used because the vibrations produce a surface polarization which results in an electric field normal to the surface equal to that component of polarization, and opposite in direction, so that the absolute value of \&* reaches a minimum. As shown by Berreman [69] and Fuchs and Kliewer [70] for a sufficiently thin film deposited on a metallic substrate the vibrations parallel to the surface (TO modes) are completely suppressed using p-polarized light. This is because 
the vibrational dipole moment of the TO modes is entirely suppressed by the response of the metal substrate underneath the film. For the films with atomic thickness (and also for the adsorbed molecules) this effect is the origin of the selection rule discussed above, since only the LO vibrations are in general the completely symmetrical modes $[67,73]$.

Although the LO and TO terminology have been successfully used to interpret vibrational properties of many bulk materials and thin films (see e.g. refs. [74-76]), the classification of vibrational modes in terms of LO and TO modes cannot be applied straightforwardly for the case of ultra-thin, monolayer films such as $\mathrm{SiO}_{2} / \mathrm{Mo}(112)$ discussed here. It is obvious that all the asymmetric Si-O-Mo stretching vibrations of the 2D-network film shown in Fig. 11 are pure LO vibrations, since they occur normal to the film surface. However, the physics of this LO vibration is completely different from that of the LO modes in the cubic crystal or thicker films because there is no long-range electric field induced by this vibration that could oppose the motion of the atoms. Indeed, there is just one Si-O-Mo bond perpendicular to the surface with metal surface below and vacuum above it, so no long-range electrostatic response is possible. Moreover, there is no TO mode associated with these vibrations since it would require $\mathrm{Si}-\mathrm{O}-\mathrm{Mo}$ bonds aligned parallel (or at least at a reasonably small angle) to the metal surface. Similarly, all combinations of the asymmetric Si-O-Si stretching vibrations are pure TO modes since they have no polarization perpendicular to the surface. The corresponding LO vibration is impossible because it would require $\mathrm{Si}-\mathrm{O}-\mathrm{Si}$ bonds perpendicular to the surface, which exist in the case of thicker or amorphous $\mathrm{SiO}_{2}$ films, that Chen et al. $[43,45]$ referred to. Only the symmetric Si-O-Si vibration modes can naively be classified as either LO and TO modes. The vibrations observed at 779 and $672 \mathrm{~cm}^{-1}$ have vibrational dipole moment component perpendicular to the surface (thus LO modes) whereas remaining four vibrations have either zero net vibrational dipole moment or it is parallel to the surface (TO modes). However, the "LO" symmetric Si-O-Si vibrations again differ from that of bulk silica materials or thicker films because no long-range electric field induced by this vibration is possible in the monolayer silica film. This analysis shows that the spectra of ultra-thin crystalline films cannot be interpreted by a straightforward comparison with bulk materials and thicker amorphous or crystalline films as done in Ref. [43]. The 
detailed insight into structure and properties of thin oxide systems can only be reached combining careful experiments and modern theoretical methods.

\section{Concluding remarks.}

Well-ordered, monolayer silica films can be prepared on a Mo(112) substrate. The analysis of the experimental results obtained using STM, XPS, UPS, IRAS and DFT calculations strongly support the model where the silica film consists of a twodimensional network of corner sharing $\left[\mathrm{SiO}_{4}\right]$ tetrahedra with one oxygen atom bonded to the Mo substrate atoms. The structure may additionally involve oxygen atoms directly adsorbed on $\mathrm{Mo}(112)$. It may well be that the "O-poor" and "O-rich" films may coexist depending on the preparation conditions. In addition, the annealing temperature is critical to the presence of the silica particles on the surface, which in turn may influence the electronic and vibrational properties of the films. These findings may explain the discrepancy between the experimental results reported in the literature.

The review shows that it is very important to use a combination of different experimental techniques applied to the same sample, in attempts to determine the atomic structure of thin oxide films where a substrate can deeply be involved in the stabilization of the oxide structure.

A critical role of theoretical studies for determining the atomic structure of the complex systems is highlighted. In particular, interpretation of the vibrational properties of the thin oxide films cannot be properly done without theoretical analysis.

\section{Acknowledgements.}

We gratefully acknowledge Junling Lu for his contribution in the experimental results used in this review. T.K.T. and S.K. thank the International Max Planck Research School (IMPRS) "Complex Surfaces in Materials Science", and J.W. and D.S. the Alexander von Humboldt Foundation for fellowships. The work was supported by Deutsche Forschungsgemeinschaft (SFB 546), and also by the EU Project GSOMEN. 
The calculations were carried out at the Norddeutscher Verbund für Hoch- und Höchstleistungsrechnen (HLRN). 


\section{References.}

1. D. W. Goodman, Surf. Rev. Lett., 2 (1995), 9.

2. C.T. Campbell, Surf. Sci. Rep., 27 (1997), 1.

3. H.-J. Freund, Angew. Chem. Int. Ed., 36 (1997), 452.

4. R. Franchy, Surf. Sci. Rep., 38 (2000), 195.

5. S.A. Chambers, Surf. Sci. Rep., 39 (2000), 105.

6. M. Bäumer, H.-J. Freund, Prog. Surf. Sci., 61 (1999), 127.

7. W. Weiss, W. Ranke, Prog. Surf. Sci., 70 (2002), 1.

8. U. Diebold, Surf. Sci. Rep., 48 (2003), 53.

9. G. Kresse, M. Schmid, E. Napetschnig, M. Shishkin, L. Köhler, P. Varga, Science, 308 (2005), 1440.

10. M. Sterrer, M. Yulikov, E. Fischbach, M. Heyde, H.-P. Rust, G. Pacchioni, T. Risse, H.-J. Freund, Angew. Chem. Int. Ed., 45 (2006), 2630.

11. D.R. Mullins, P.V. Radulovic, S.H. Overbury, Surf. Sci., 429 (1999), 186.

12. J. Schoiswohl, M. Sock, S. Surnev, M.G. Ramsey, F.P. Netzer, G. Kresse, J.N. Andersen, Surf. Sci., 555 (2004), 101.

13. J.W. He, X. Xu, J.S. Corneille, D.W. Goodman, Surf.Sci., 279 (1992), 119.

14. X. Xu, D.W. Goodman, Surf. Sci., 282 (1993), 323.

15. T. Schroeder, M. Adelt, B. Richter, M. Naschitzki, M. Bäumer, H.-J. Freund, Surf. Rev. Lett. 7,7 (2000).

16. T. Schroeder, M. Adelt, B. Richter, M. Naschitzki, M. Bäumer, H.-J. Freund, Microelectr. Rel. 40 (2000), 841.

17. T. Schroeder, A. Hammoudeh, M. Pykavy, N. Magg, M. Adelt, M. Bäumer, H.-J. Freund, Solid State Electronics, 45 (2001), 1471.

18. T. Schroeder, J. B. Giorgi, M. Bäumer, H.-J. Freund, Phys. Rev. B 66 (2002), 165422.

19. J.B. Giorgi, T. Schroeder, M. Bäumer, H.-J. Freund, Surf. Sci. 498 (2002), L71.

20. A.K. Santra, B.K. Min, D.W. Goodman, Surf. Sci. 515 (2002) L475.

21. B.K. Min, A.K. Santra, D.W. Goodman, J. Vac. Sci. Technol. B 21 (2003), 2319.

22. Y. D. Kim, T. Wei, D.W. Goodman, Langmuir 19 (2003), 354.

23. B. K. Min, A. K. Santra, D. W. Goodman, Catal. Today 85 (2003), 113.

24. Y. D. Kim, T. Wei, J. Stulz, D.W. Goodman, Langmuir 19 (2003), 1140.

25. E. Ozensoy, B.K. Min, A.K. Santra, D.W. Goodman, J. Phys. Chem. B 108 (2004), 4351.

26. S. Wendt, M. Frerichs, T. Wei, M. Chen, V. Kempter, D.W. Goodman, Surf. Sci. 565 (2004) 107.

27. D. Ricci, G. Pacchioni, Phys. Rev. B 69 (2004), 161307.

28. M. Chen, A.K. Santra, D.W. Goodman, Phys. Rev. B 69 (2004), 155404.

29. B.K. Min, W.T. Wallace, D.W. Goodman, J. Phys. Chem. B 108 (2004), 14609.

30. B.K. Min, W.T. Wallace, A.K. Santra, D.W. Goodman, J. Phys. Chem. B., 108 (2004), 16339.

31. W.T. Wallace, B.K. Min, D.W. Goodman, J. Mol. Catal. A 228 (2005), 3.

32. J. Weissenrieder, S. Kaya, J.-L. Lu, H.-J. Gao, S. Shaikhutdinov, H.-J. Freund, M. M. Sierka, T. K. Todorova, J. Sauer, Phys. Rev. Lett. 95 (2005), 076103.

33. L. Giordano, D. Ricci, G. Pacchioni, P. Ugliengo, Surf. Sci. 584 (2005), 225.

34. I.N. Yakovkin, Surf. Rev. Lett., 12 (2005), 449.

35. S. Wendt, E. Ozensoy, T. Wei, M. Frerichsr, Y. Cai, M. Chen, D.W. Goodman, Phys. Rev. B 72 (2005), 115409 . 
36. T.K. Todorova, M. Sierka, J. Sauer, S. Kaya, J. Weissenrieder, J.-L. Lu, H.-J. Gao, S. Shaikhutdinov, H.-J. Freund, Phys. Rev. B 73 (2006), 165414.

37. M. Sierka, T.K. Todorova, S. Kaya, D. Stacchiola, J. Weissenrieder, J.-L. Lu, H.-J. Gao, S. Shaikhutdinov, H.-J. Freund, J. Sauer, Chem. Phys. Lett. 424 (2006), 115.

38. B.K. Min, W.T. Wallace, D.W. Goodman, Surf. Sci., 600 (2006), L7.

39. L. Giordano, A. Del Vitto, G. Pacchioni, J. Chem Phys., 124 (2006), 034701.

40. J.-L. Lu, S. Kaya, J. Weissenrieder, T.K. Todorova, M. Sierka, J. Sauer, H.-J. Gao, S. Shaikhutdinov, H.-J. Freund, Surf. Sci., 600 (2006) L164.

41. J.-L. Lu, S. Kaya, J. Weissenrieder, H.-J. Gao, S. Shaikhutdinov, H.-J. Freund, Surf. Sci., 600 (2006), L153.

42. U. Martinez, L. Giordano, G. Pacchioni, J. Chem Phys. B 110 (2006), 17015.

43. M. Chen, D.W. Goodman, Surf. Sci., 600 (2006), L255.

44. L. Giordano, D. Ricci, G. Pacchioni, P. Ugliendo, Surf. Sci. 601 (2007), 588.

45. M. Chen, D.W. Goodman, Surf. Sci. 601 (2007), 591.

46. S. Shaikhutdinov, M. Ritter, W. Weiss, 62 (2000), 7535.

47. F. Sedona, S. Agnoli, G. Granozzi, J. Phys. Chem. B 110 (2006), 15359.

48. D.E. Starr, F.M.T. Mendes, J. Middeke, R.-P.Blum, H. Niehus, D. Lahav, S. Guimond, A. Uhl, T. Kluener, M. Schmal, H. Kuhlenbeck, S. Shaikhutdinov, H.-J. Freund, Surf. Sci., 599 (2005), 14.

49. J. F. Moulder, W. F. Stickle, P. E. Sobol, K. D. Bomben, Handbook of X-ray Photoelectron Spectroscopy, Perkin-Elmer Corporation USA, 1992.

50. F.J. Grunthaner, P.J. Grunthaner, R. Vasguez, B. Lewis, J. Maserjian, A. Madhukar, J. Vac. Sci. Technol. 16 (1979), 1443.

51. T. Schroeder, J. Zegenhagen, N. Magg, B. Immaraporn, H.-J. Freund, Surf. Sci. 552 (2004), 85.

52. T.H. Di Stefano, D.E. Eastman, Phys. Rev. Lett. 27 (1971), 1560.

53. B. Fischer, R.A. Pollak, T.H. Di Stefano, W.D. Grobman, Phys. Rev. B 15 (1977), 3193.

54. J. Stöhr, NEXAFS spectroscopy, Springer-Verlag, Berlin (1992).

55. J.G. Chen, Surf. Sci. Rep. 30 (1997), 1.

56. M. Sing, R. Neudert, H. von Lips, M.S. Golden, et al., Phys. Rev. B 60 (1999), 8661.

57. M. Cavalleri, K. Hermann, S. Guimond, Y. Romanyshyn, H. Kuhlenbeck, H.-J. Freund, submitted

58. M. Taillefumier, D. Cabaret, A.-M. Flank, F. Mauri, Phys. Rev. B 66 (2002), 195107.

59. L.A.J. Garvie, P. Rez, J.R. Alvarez, P.R. Buseck, A.J. Craven, R. Brydson, Am. Mineral. 85 (2000), 732.

60. I. Tanaka, J. Kawai, H. Adachi, Phys. Rev. B 52 (1995), 11733.

61. A.V. Soldatov, M. Kasrai, G.M. Bancroft, Solid State Commun. 115 (2000), 687.

62. S.-D. Mo, W.Y. Ching, Appl. Phys. Lett 78 (2001), 3809.

63. K. Reuter, M. Scheffler, Phys. Rev. B 65 (2002), 035406.

64. F.M. Hoffman, Surf. Sci. Rep. 3 (1983), 107.

65. N. Sheppard, J. Erkelens, Appl. Spectroscopy 38 (1984), 471.

66. A. M. Bradshaw, N. V. Richardson, Pure \& Appl. Chem. 68 (1996), 457.

67. H. Ibach, D. L. Mils, Electron Energy Loss Spectroscopy and Surface Vibrations, Academic Press, New York, 1982.

68. H. Ibach, Surf. Sci. 66 (1977), 56.

69. D.W. Berreman, Phys. Rev. 130 (1963), 2193.

70. R. Fuchs, K. L. Kliewer, Phys. Rev. 140 (1965), 2076. 
71. M. Born, K. Huang, Dynamical Theory of Crystal Lattices, Oxford University Press 1966, Oxford.

72. H. Böttger, Principles of the Theory of Lattice Dynamics, Physik-Werlag 1983, Weinheim.

73. H. A. Pearce, N. Sheppard, Surface Sci. 59 (1976) 205.

74. F.L. Galeener, G. Lucovsky, Phys. Rev. Lett. 37 (1976) 1474.

75. B. Harbecke, B. Heinz, P. Grosse, Appl. Phys. A 38 (1985) 263.

76. P. Grosse, B. Harbecke, B. Heinz, R. Meyer, M. Offenberg, Appl. Phys. A 39 (1986) 257. 
Table 1. Calculated harmonic frequencies (in $\mathrm{cm}^{-1}$ ) of vibrational modes for the 2D-network model and transformation properties of the normal modes with respect to the eight symmetry elements of the $\mathrm{cmm}$ two-dimensional space symmetry group (E - identity, $\mathrm{C}_{2}$ - twofold symmetry axis, $\sigma(x z)$ symmetry plane, $\sigma^{\prime}(x z)$ glide plane, $\tau$ - translational period).

\begin{tabular}{|c|c|c|c|c|c|c|c|c|}
\hline Modes $\left(\mathrm{cm}^{-1}\right)$ & $\mathrm{E}$ & $\mathrm{C}_{2}$ & $\sigma(x z)$ & $\sigma(y z)$ & $1 / 2 \tau$ & $\mathrm{C}_{2}^{\prime}$ & $\sigma^{\prime}(x z)$ & $\sigma^{\prime}(y z)$ \\
\hline \multicolumn{9}{|c|}{ Asymmetric Si-O-Si } \\
\hline$v_{1}=1195$ & 1 & -1 & -1 & 1 & -1 & 1 & 1 & -1 \\
\hline$v_{2}=1137$ & 1 & -1 & -1 & 1 & 1 & -1 & -1 & 1 \\
\hline$v_{3}=1092$ & 1 & 1 & 1 & 1 & -1 & -1 & -1 & -1 \\
\hline$v_{4}=1018$ & 1 & -1 & -1 & 1 & 1 & -1 & -1 & 1 \\
\hline$v_{5}=1010$ & 1 & -1 & 1 & -1 & 1 & -1 & 1 & -1 \\
\hline$v_{6}=1008$ & 1 & 1 & -1 & -1 & -1 & -1 & 1 & 1 \\
\hline \multicolumn{9}{|c|}{ Asymmetric Si-O-Mo } \\
\hline$v_{1}=1061$ & 1 & 1 & 1 & 1 & 1 & 1 & 1 & 1 \\
\hline$v_{2}=912$ & 1 & -1 & -1 & 1 & -1 & 1 & 1 & -1 \\
\hline$v_{3}=881$ & 1 & 1 & 1 & 1 & -1 & -1 & -1 & -1 \\
\hline$v_{4}=863$ & 1 & -1 & -1 & 1 & 1 & -1 & -1 & 1 \\
\hline \multicolumn{9}{|l|}{ Symmetric Si-O-Si } \\
\hline$v_{1}=821$ & 1 & -1 & 1 & -1 & -1 & 1 & -1 & 1 \\
\hline$v_{2}=817$ & 1 & 1 & -1 & -1 & 1 & 1 & -1 & -1 \\
\hline$v_{3}=779$ & 1 & 1 & 1 & 1 & 1 & 1 & 1 & 1 \\
\hline$v_{4}=695$ & 1 & -1 & -1 & 1 & -1 & 1 & 1 & -1 \\
\hline$v_{5}=676$ & 1 & 1 & 1 & 1 & -1 & -1 & -1 & -1 \\
\hline$v_{6}=672$ & 1 & 1 & 1 & 1 & 1 & 1 & 1 & 1 \\
\hline
\end{tabular}


Figures.
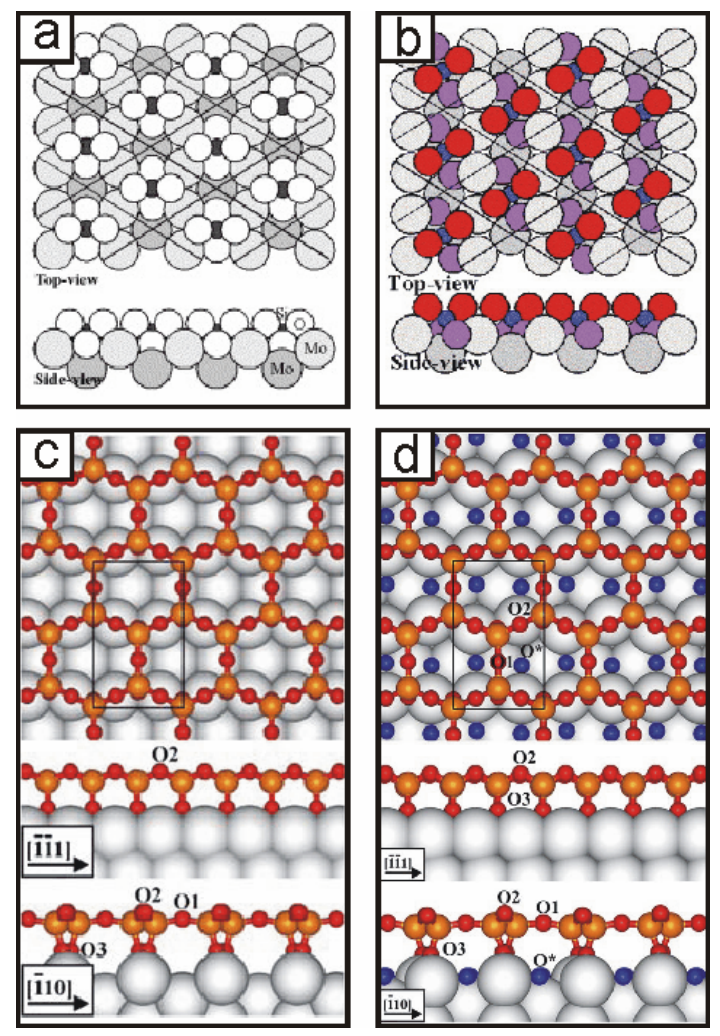

Figure 1. Different models proposed for the monolayer silica film grown on $\operatorname{Mo}(112)$. (a,b) "Cluster models" as suggested in [28] (a) and [43] (b). Shown in the (c) and (d) are "2D-network" models for the "O-poor" and "O-rich" films, respectively [32,37]. 


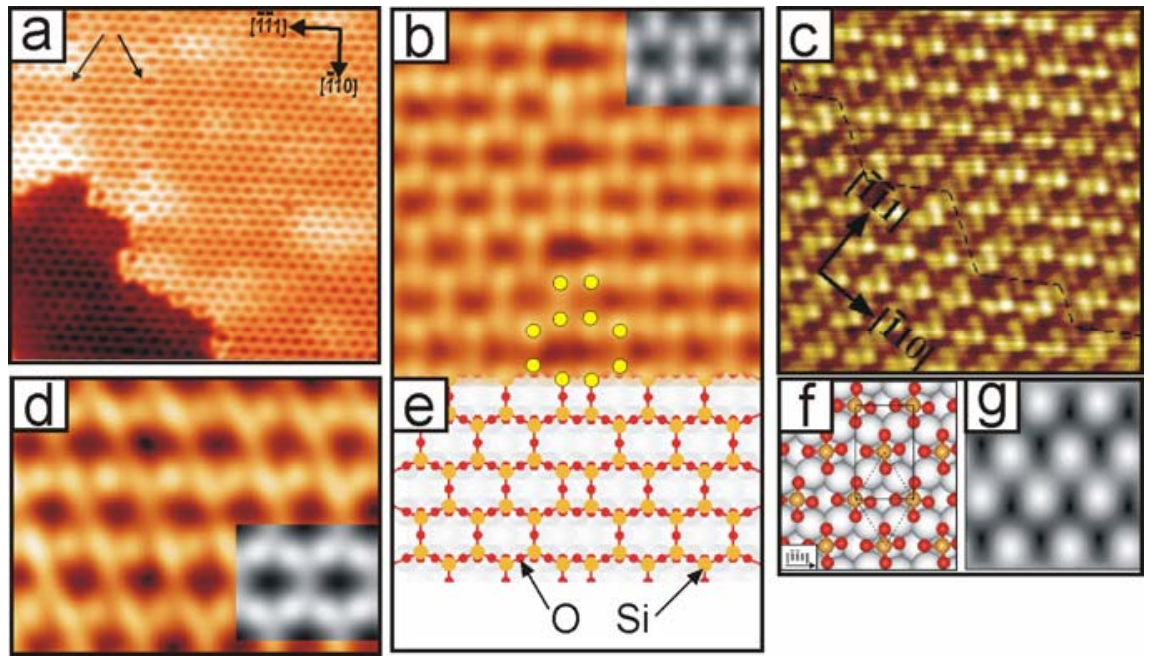

Figure 2. Large-scale (a) and atomically resolved (b,d) STM images of the "O-poor" silica films together with the image simulation of the model shown in Fig. 1c. (b) High-resolution image of antiphase domain boundaries, indicated by the arrows in (a), is superimposed with the APDB model shown in (e). The atomic protrusions seen in (b) and (d) represent the $\mathrm{Si}$ and $\mathrm{O}$ atoms, respectively. The crystal directions shown in (a) are applied to all figures except (c). The STM image reported in [43] is shown in (c), for comparison. DFT-optimized geometry and simulated STM image for the cluster models are shown in (f) and (g). The size and tunnelling parameters are: (a) $14 \times 14 \mathrm{~nm}^{2} ; \mathrm{V}=1.3 \mathrm{~V}, \mathrm{I}=0.45 \mathrm{nA}$; (b) $3.2 \times 3.2 \mathrm{~nm}^{2} ; 1.2 \mathrm{~V}, 0.35$ $\mathrm{nA}$; (c) $5.5 \times 5.5 \mathrm{~nm}^{2}$; $-3 \mathrm{~V}, 0.3 \mathrm{nA}$; (d) $3 \times 2.25 \mathrm{~nm}^{2}$; $0.65 \mathrm{~V}, 0.75 \mathrm{nA}$.

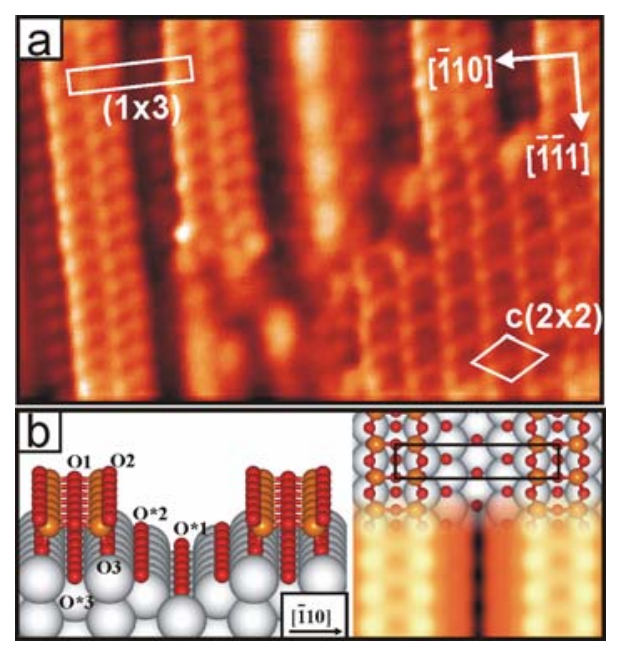

Figure 3. One dimensional silica stripes observed at sub-monolayer silica coverage. STM image (a) and the model (b) of the stripes superimposed with the simulated STM image. The size is $6.5 \times 4.5 \mathrm{~nm}^{2}$, tunnelling parameters are $\mathrm{V}=-0.5 \mathrm{~V}, \mathrm{I}=0.4 \mathrm{nA}$. 


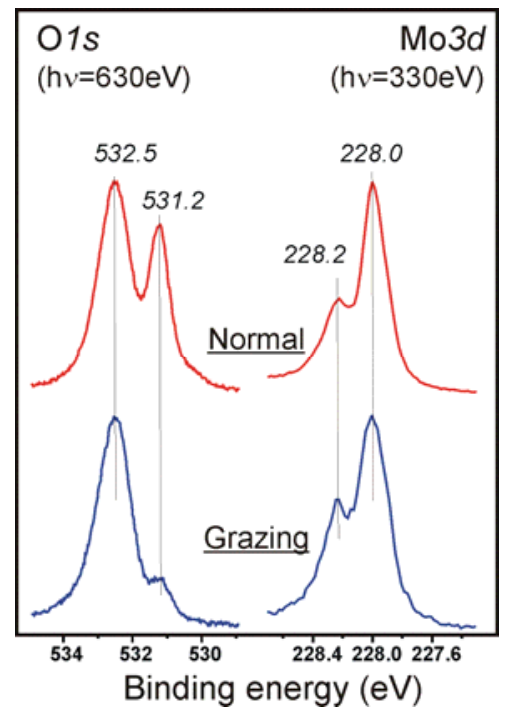

Figure 4. The $\mathrm{O} 1 \mathrm{~s}$ and Mo3d photoelectron spectra using synchrotron radiation observed for the "O-poor" film at normal and grazing $\left(80^{\circ}\right)$ emissions. The light energies $(\mathrm{h} v)$ are indicated.

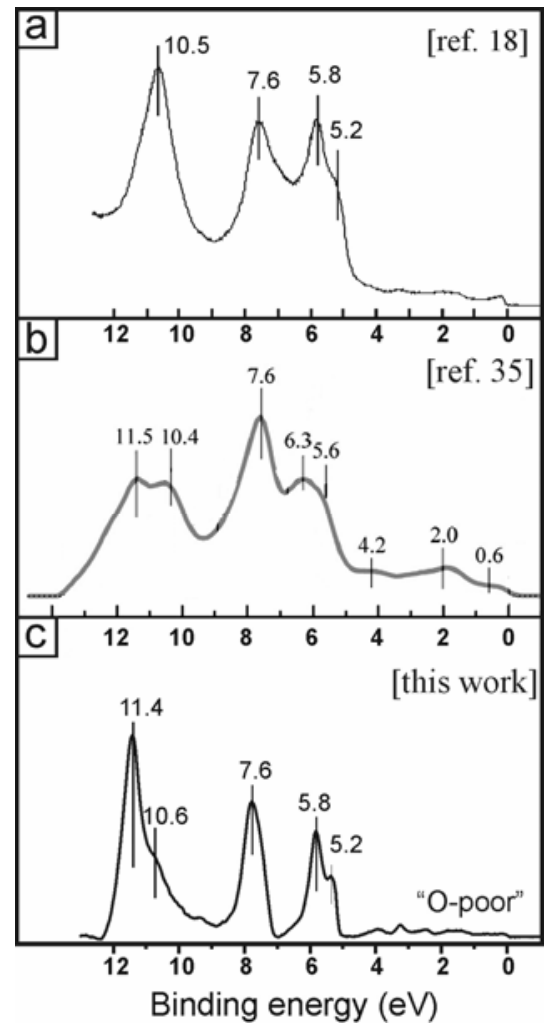

Figure 5. (a) Ultraviolet photoelectron spectrum reported by Schroeder et al. [18]. (b) UP-spectrum from the ref. [35] for the $\sim 1.2 \mathrm{ML}$ film. (c) The UP-spectrum (He I) of the "O-poor" monolayer film observed in the present work. 


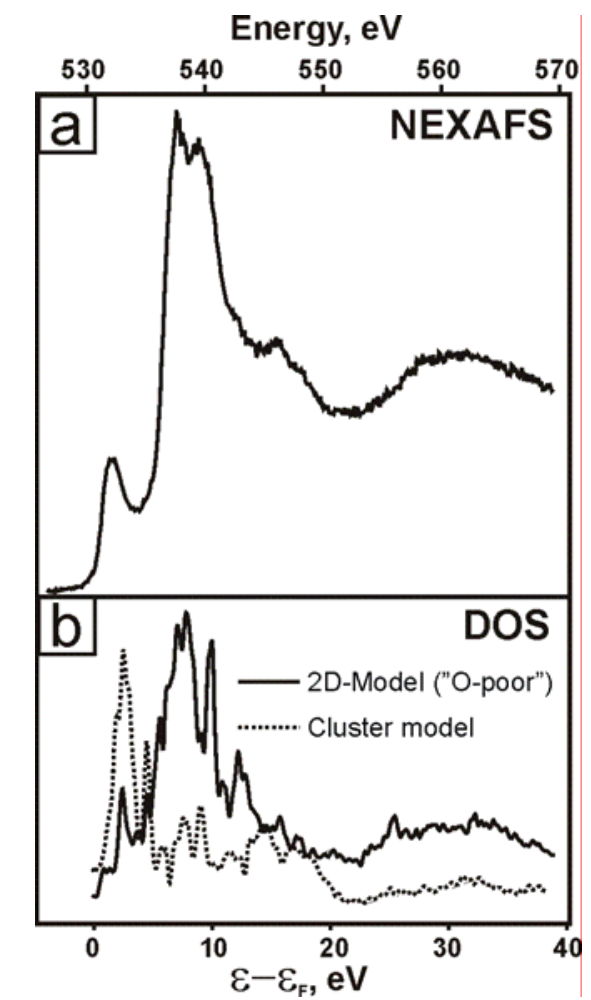

Figure 6. (a) Oxygen K-edge NEXAFS spectrum of the silica film. The E vector of the synchrotron light is parallel to the [1 10] direction. (b) Calculated density of unoccupied states above the Fermi level for two different models discussed.

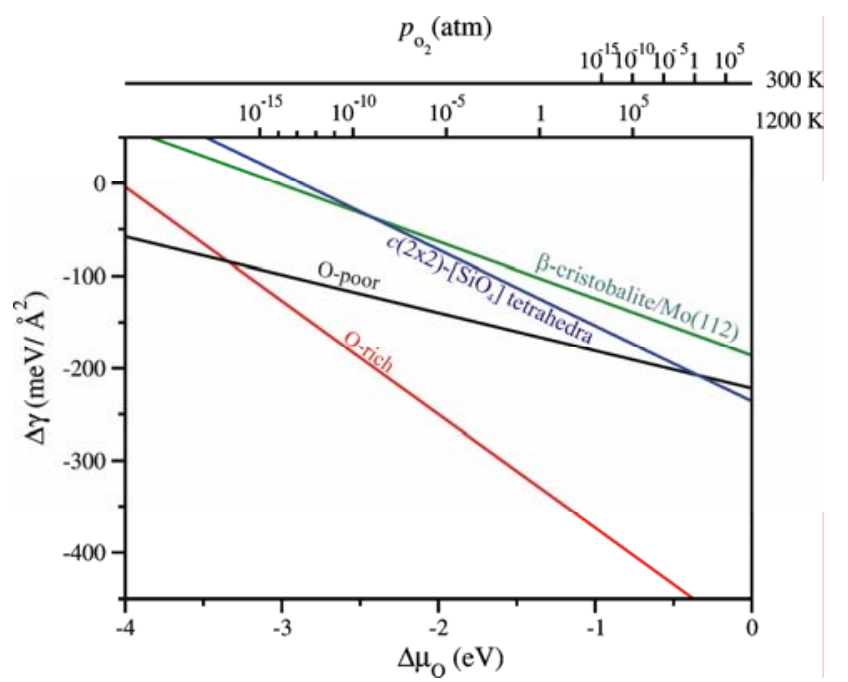

Figure 7. Stability plot of the different models discussed in this paper (see the text). The upper scale indicates the $\mathrm{O}_{2}$ pressures at 300 and $1000 \mathrm{~K}$ corresponding to the oxygen chemical potential. 


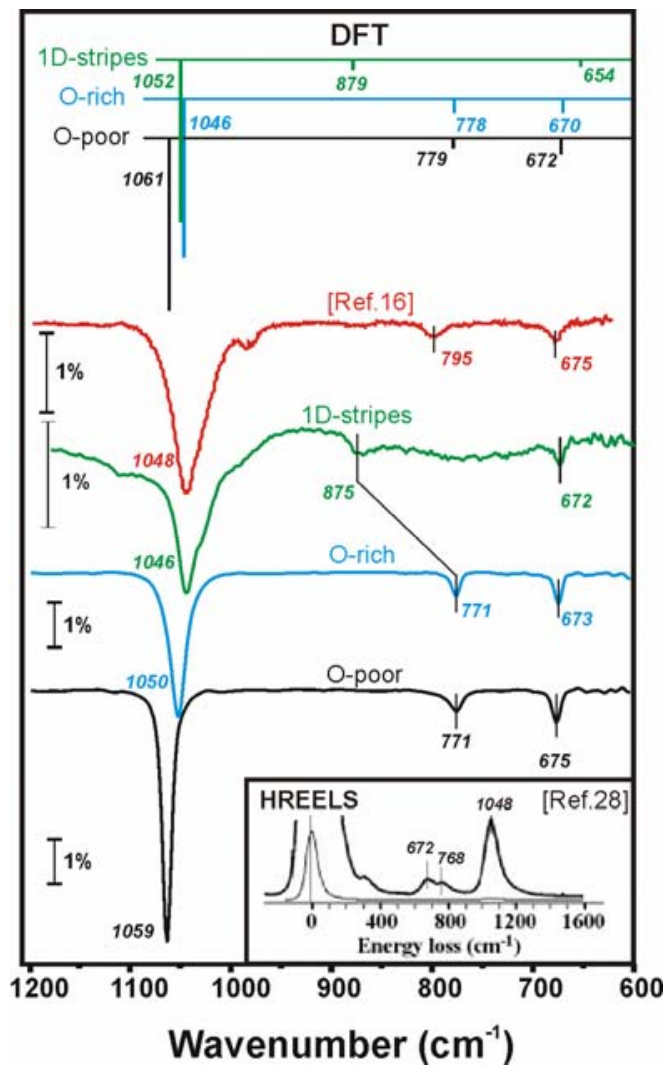

Figure 8. IRA-spectra of the "O-poor", "O-rich" films and silica stripes $[33,36,37,40]$. The spectrum reported by the Schroeder et al. [18] is shown for comparison. Shown in the inset is the HREEL- spectrum reported by Chen et al. [28]. The frequencies of the IRAS active modes calculated for the 2D-network model are shown as the bars with the height proportional to its intensity. (For paper online: the same colour code is used for calculated and experimental results). 


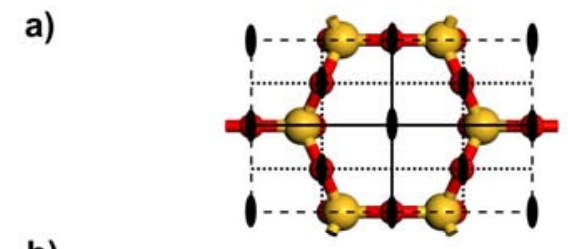

b)

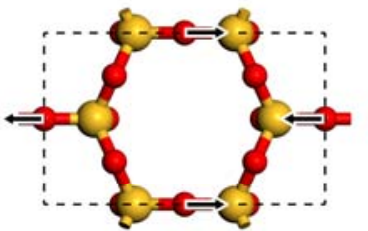

1195

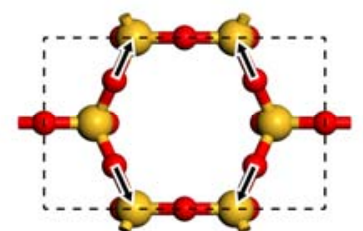

1092

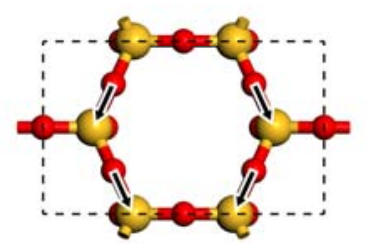

1010

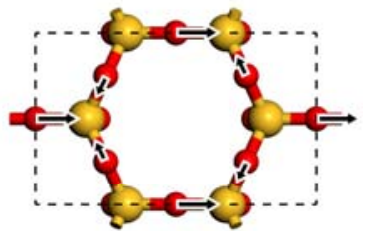

1137

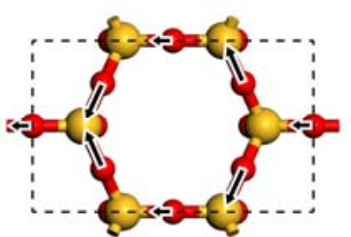

1018

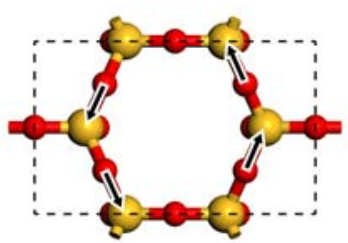

1008

Figure 9. (a) Symmetry elements of the $\mathrm{cmm}$ symmetric surface unit cell of the 2D-network model of the $\mathrm{SiO}_{2} / \mathrm{Mo}(112)$ film (top view). Large yellow balls are $\mathrm{Si}$, small red balls are O. The ellipsoids represent the twofold symmetry axes, solid lines - the symmetry planes and dotted lines - the glide planes; (b) Schematic representation of normal vibrational modes involving couplings of the asymmetric Si-O-Si vibrations along with the calculated frequencies $\left(\mathrm{cm}^{-1}\right)$. 


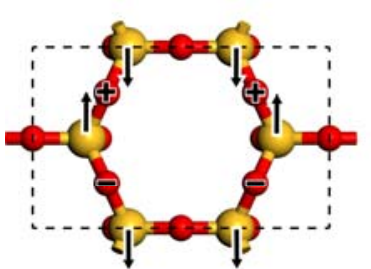

821

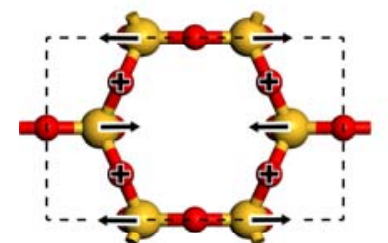

779

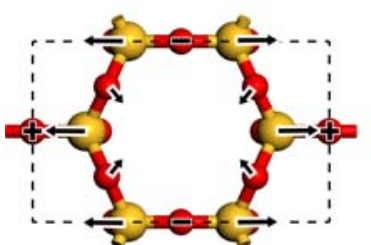

676

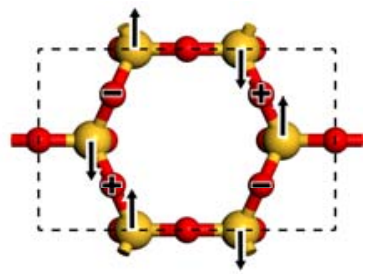

817

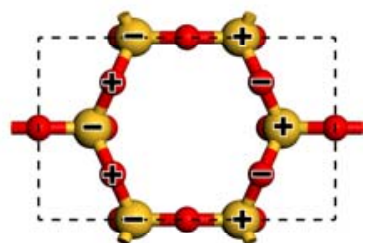

695

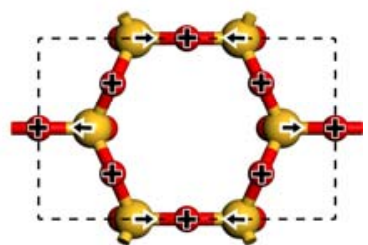

672

Figure 10. Schematic representation of normal vibrational modes involving couplings of symmetric Si-OSi vibrations (top view) along with the calculated frequencies $\left(\mathrm{cm}^{-1}\right)$. The plus and minus signs indicate atomic motions perpendicular to the surface.

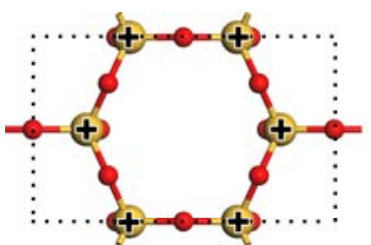

1061

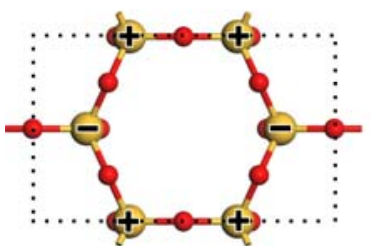

881

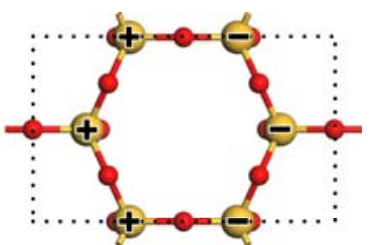

912

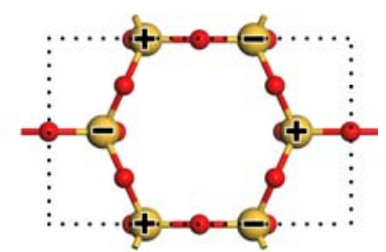

863

Figure 11. Schematic representation of normal vibrational modes involving couplings of Si-O-Mo stretching vibrations (top view) along with the calculated frequencies $\left(\mathrm{cm}^{-1}\right)$. The plus and minus signs indicate atomic motions perpendicular to the surface. 